\title{
Marianne Soff
}

\section{Demokratie als Gute Ordnung des Zusammenlebens}

\section{Erziehung zu Toleranz und Mitverantwortung in Freiheit bei Kurt Lewin und Wolfgang Metzger}

\section{Einleitung}

„Die liberale Demokratie und das politische und normative Projekt des Westens, sie stehen unter Beschuss“ konstatierte Joachim Gauck, der scheidende deutsche Bundespräsident, in seiner Abschiedsrede am 18. Januar 2017 (vgl. Edler \& Schnack, 2017, S. 6). Die gegenwärtigen weltweiten Krisen führen auch in Europa zu spürbaren politischen und gesellschaftlichen Veränderungen. Zugleich kommt es zu einer zunehmenden Ausbreitung von extremen und intoleranten Meinungen, mitunter verbunden mit (entschiedener oder gedankenloser) Ablehnung demokratischer Gesellschaftsstrukturen. In dieser Lage ist es erforderlich, dass in Schulen und anderen pädagogischen Einrichtungen Demokratie und Menschenrechte thematisiert und in aktiver Beziehungsgestaltung „gelebt“ werden. Für Lehrkräfte, die sich dafür - neben der Bewältigung ihrer tagesaktuellen Anforderungen - um die Weiter-Entwicklung einer eigenen kritischen und reflexiven Haltung bemühen, stellt sich dringlich die Frage nach psychologischen Grundlagen und Konzepten für die Vorbereitung von Kindern und Jugendlichen auf ein mit-menschliches Zusammenleben. Dazu soll die folgende (Wieder-)Begegnung mit Werkauszügen zweier „Klassiker der Psychologie“ beitragen, die als Diskussionsgrundlage für die Beschäftigung mit Demokratie, Freiheit und Toleranz gedacht ist.

Die Schriften von Kurt Lewin und Wolfgang Metzger zu Fragen des menschlichen Zusammenlebens in Gruppen und zum gesellschaftlichen Miteinander sind mittlerweile Jahrzehnte alt, aber zugleich erstaunlich aktuell. $\mathrm{Zu}$ berücksichtigen ist, dass auch ihre Entstehungszeiten um Jahre auseinander liegen und die Umstände ihrer Entstehung verschieden waren: Kurt Lewin (1890-1947) schrieb die im Folgenden zitierten Texte als ein der psychologischen Theoriebildung und der sozialen Praxis gleichermaßen verpflichteter Forscher in den 1930er und 1940er Jahren nach seiner Emigration in die USA. Die hier verwendeten Texte des Münsteraner Hochschullehrers für Psychologie und Pädagogik Wolfgang Metzger (1899-1979) wurden erst in den 1970er Jahren in der damaligen Bundesrepublik Deutschland verfasst. Trotz mancher Unterschiede in der Schwerpunktsetzung ihrer Argumentation erscheint es 
stimmig, sie als gemeinsamen Beitrag von Gestalttheorie und Feldtheorie zu Demokratie und Toleranz anzusehen - vor dem Hintergrund eines Menschenbildes, das soziale und ethische Aspekte ausdrücklich betont.

\section{Grundlegendes: Die Bedeutung von Gruppen im Leben des Einzelnen}

Menschen werden in Gestalttheorie und Feldtheorie als von Anfang an soziale Wesen gesehen, die sozialen Bedürfnisse motivieren und moderieren grundsätzlich menschliches Verhalten und Erleben. Damit zugleich werden die Gruppen, in denen Menschen zusammenleben, zum wichtigen Thema und Untersuchungsgegenstand auch der Psychologie ${ }^{1}$.

Kurt Lewin, dessen feldtheoretische Begriffe und Methoden auf die Gestalttheorie zurückgehen (vgl. Horkheimer, 1953, S. 8), argumentiert in seinen Begriffsbestimmungen zum Begriff Gruppe (nahezu gleichlautend 1939b, S. $114 \mathrm{f}$ und 1939c, S. 182f) explizit gestaltpsychologisch:

„Die Definition des Begriffs, Gruppe' hat eine etwas chaotische Geschichte. Der Ausdruck ist mit philosophischen und metaphysischen Überlegungen verflochten. Es war einer der Hauptdiskussionspunkte, ob die Gruppe eine Gruppenseele habe oder nicht und ob sie darum eine Entität über dem Individuum und außerhalb von ihm sei. (...)

Für einen Psychologen, der die historische Entwicklung der Begriffe, Ganzheit' oder Gestalt in der Psychologie verfolgt hat, klingt das meiste aus der Argumentation über die Gruppenseele merkwürdig vertraut. (...) Noch vor kurzer Zeit (in der frühen Gestaltpsychologie) wurde häufig die Feststellung getroffen, daß ,das Ganze mehr ist als die Summe seiner Teile'. Heute kann diese Formulierung kaum als adäquat betrachtet werden. Das Ganze ist nicht, mehr' als die Summe seiner Teile, sondern es hat andere Eigenschaften. Der Satz sollte lauten: ,Das Ganze ist etwas anderes als die Summe seiner Teile.' Mit anderen Worten, es besteht keine Wertüberlegenheit des Ganzen. Beide, Ganzes und Teile, sind gleichermaßen wirklich. Andererseits hat das Ganze bestimmte eigene Eigenschaften, die nur ihm zukommen. Diese Feststellung hat ihren magischen Schimmer verloren und wurde eine einfache wissenschaftliche Tatsache, seit man entdeckte, daß für physikalische Ganze und Teile Gleiches gilt. Darüberhinaus anerkennt die Psychologie heute, daß es Ganzheiten aller Abstufungen dynamischer Einheitlichkeit gibt: im einen Extrem gibt es Aggregate unabhängiger Gegenstände, gefolgt von Ganzen mit geringem Grad an Einheitlichkeit, von mittleren Graden an Einheitlichkeit und schließlich im anderen Extrem von so hohem Grad an Einheitlichkeit, daß es kaum angemessen ist, von Teilen zu sprechen." (Lewin, 1939c, S. 182f, Kursivhervorhebungen MS)

\footnotetext{
${ }^{1}$ Übrigens ist mittlerweile die Bedeutung von Gruppen unter dem Begriff "social brain" sogar zum neuropsychologischen Untersuchungsgegenstand geworden (vgl. Dunbar, 1998, 2009).
} 
Zusammenfassend kann man im Sinn der Schriften Lewins und Metzgers eine Gruppe als dynamisches, überindividuelles Ganzes definieren, mit Gruppeneigenschaften, die nicht mit den Eigenschaften der einzelnen Gruppen-Mitglieder identisch sind, und mit Interdependenz der Mitglieder. Eine Gruppe stellt somit ein Beispiel für eine Gestalt mit Struktur- und Wesenseigenschaften dar. Eine wichtige Wesenseigenschaft ist die Gruppenatmosphäre, die etwa "freundlich“, „offen “ und „aufgabenorientiert" oder auch „angespannt“ und „konkurrenzorientiert" sein kann. Strukturen sind im Fall von Gruppen als dynamische Strukturen zu verstehen, die man mit Metzger auch als die sich herausbildenden „Ordnungen des Zusammenlebens“ (vgl. Metzger, 1976a, S. 92f) in einer Gruppe bezeichnen kann. Diese Ordnungen können grundsätzlich entweder mittels formaler Regeln und Verordnungen verbindlich festgelegt werden oder sich in einem „natürlichen Wechselspiel der Kräfte“ im Rahmen der Selbstorganisation der Gruppe immer wieder neu ergeben (vgl. Metzger, 1975b, S. 22). Der Grad an freier Selbstorganisation in Gruppen variiert dabei beträchtlich. Beispiele für solche (in einzelnen Gruppen sehr verschiedenen) strukturellen Eigenschaften sind etwa die Kommunikations- und Interaktionswege innerhalb einer Gruppe, die Art der Gruppenleitung und der Hierarchiebildung, die Rollen und Zuständigkeiten der einzelnen Mitglieder innerhalb der Gruppe. Wie Lewin in den Experimenten mit Lippitt und White gezeigt hat, beeinflusst insbesondere die Art der Ausübung der Führungsfunktion die Gruppenatmosphäre erheblich, das bedeutet, es besteht auch in Gruppen ein enger Zusammenhang von Struktur- und Wesenseigenschaften (vgl. Metzger, 2001, S.65f).

In seiner Definition einer „gut organisierten Gruppe mit hohem Einheitlichkeitsgrad" betont Lewin das Merkmal der Interdependenz der Gruppenmitglieder als konstituierenden Faktor. Nicht auf die Ähnlichkeit oder gar Gleichheit (Homogenität) der Mitglieder komme es bei einer prägnanten Gruppe an, sondern auf ihre wechselseitige Abhängigkeit:

„Faßt man die Gruppe als dynamisches Ganzes auf, dann sollte eine Definition der Gruppe auf die wechselseitige Abhängigkeit ihrer Glieder abstellen (oder, besser, auf die Teilbereiche der Gruppe). Mir scheint es ziemlich wichtig, diesen Punkt zu betonen; denn viele Definitionen der Gruppe benutzen als konstituierenden Faktor die Ähnlichkeit der Gruppenangehörigen anstatt ihre dynamische Interdependenz. Die Gruppe wird zum Beispiel häufig definiert als aus einer Anzahl bestimmter Personen zusammengesetzt, die bestimmte Ähnlichkeiten, besonders Ähnlichkeiten von Haltungen, aufweisen. (...) Es ist sehr wohl möglich, daß eine Anzahl Personen bestimmte Ähnlichkeiten haben - beispielsweise das Geschlecht, die Rasse, die ökonomische Stellung, die Haltungen -, ohne eine Gruppe im Sinne wechselseitig abhängiger Teile eines sozialen Ganzen zu sein. (...) 
Andererseits braucht eine Gruppe nicht unbedingt aus Angehörigen zu bestehen, die eine große Ähnlichkeit aufweisen. Tatsächlich gilt für soziale Gruppen wie für jedes Ganze irgendeines Gegenstandsgebietes, daß ein Ganzes mit sehr hohem Grad an Einheitlichkeit sehr unähnliche Teile enthalten kann. Zweifellos zeigen beispielsweise Mann, Frau und Säugling innerhalb einer Familie weit größere Unähnlichkeit als jeder Angehörige dieser Gruppe mit anderen Individuen (Säuglingen, Männern, Frauen) außerhalb der Gruppe. Für gut organisierte Gruppen mit hohem Einheitlichkeitsgrad ist es typisch, daß sie eine Anzahl von Angehörigen umfassen, die verschieden sind und verschiedene Funktionen innerhalb des Ganzen haben. Nicht die Ähnlichkeit, sondern eine gewisse Interdependenz ihrer Glieder konstituiert eine Gruppe.“ (Lewin, 1939c, S. 183f.)

Interessant ist, dass Lewin in der gleichen Arbeit (1939c, S. 184) auch das - von Metzger als Grundmerkmal angesehene - Zugehörigkeitsgefühl in seiner Definition von Gruppe als „eine Form von Ähnlichkeit“ bezeichnet, das nur dann ein Kriterium für eine Gruppe ist, wenn es Interdependenz bewirkt. Dies kritisiert Metzger Jahre später als „Verwechslung“ Lewins, mit dem Argument, dass das Zugehörigkeitsgefühl selbst, das „Teil-Sein“, ein unmittelbarer „alltäglicher Wahrnehmungssachverhalt“ sei (Metzger, 1975c, S. 223).

Auch für Metzger ist aber „die - prüfbare - wechselseitige Abhängigkeit mit Recht das Grundmerkmal“ einer Gruppe (Metzger, 1975c, S. 224). Seine Merkmalssammlung dazu, woran man erkennt, „daß ein wahrgenommener Mensch - auch die eigene Person - Mitglied einer bestimmten Gruppe ist“ (ebd.), enthält an zweiter Stelle das Zugehörigkeitsgefühl und umfasst mehrere weitere Merkmale, die an Lewin und Marrow (1969) orientiert sind: das Zusammenhalten (cohesiveness, das Bedürfnis zusammen zu sein); die Übernahme des Gruppen-Kodex (Anerkennung und Befolgung der in der Gruppe herrschenden Verhaltensregeln einschließlich der Sprache); die verstärkte gegenseitige Offenheit im Innern und eine stärkere Abgrenzung nach außen; schließlich - mit Blick auf die Beobachtungen von Spitz und Bowlby - erwähnt Metzger noch Trennungs-, Verlassenheits- und Ausstoßungsreaktionen, nämlich „Störungen der Stimmung, der Tätigkeitsbereitschaft, der Gesundheit, der körperlichen und geistigen Entwicklung“" (ebd. 1975c, S. 224), die bei Trennungen von der Gruppe vor allem bei Kindern auftreten.

Unter günstigen Umständen treten nach Metzger (1975b) auch in der Ordnung des Zusammenlebens einer Gruppe Prägnanzzustände auf, die man erkennen kann,

„wenn die Mitglieder der Gruppe sich in ihr wohlfühlen, wenn sie lieber räumlich in ihr vereinigt als allein sind, wenn sie friedlich miteinander auskommen, wenn es ihnen gelingt, ohne Sonderansprüche Einzelner reibungslos zusammenzuarbeiten, wenn sie sich aufeinander verlassen können, wenn sie bereit sind, etwa auftretende Meinungsverschiedenheiten oder einander widerstreitende Wünsche ohne Übervorteilung des 
einen oder anderen aufzulösen und bei Nöten oder Gefahren füreinander einzustehen." (Metzger, 1975b, S. 22f)

Will man die Bedeutung von Gruppen im Leben des Individuums psychologisch korrekt ausloten, so muss betont werden, dass jeder Mensch nicht nur einer einzigen Gruppe angehört. Das trifft schon für Kinder zu, mehr noch für Jugendliche und für Erwachsene. Lewin hat das 1935 klar herausgearbeitet:

„Einer der wichtigsten Bestandteile des Bodens, auf dem der einzelne Mensch steht, ist die soziale Gruppe, zu der er "gehört“. In dem Falle eines Kindes, das in einer Familie aufwächst, bildet die Familiengruppe oft seinen hauptsächlichen Boden. Wir wissen, daß eine Unsicherheit des Hintergrundes während der Kindheit zu einer Unsicherheit des Erwachsenen führen kann. Für ein Kind bedeutet es im allgemeinen einen ernsten Konflikt, sich über seine Zugehörigkeit oder Nichtzugehörigkeit zu einer Gruppe nicht im klaren zu sein.

Eines der grundlegenden Kennzeichen für Zugehörigkeit besteht darin, daß der gleiche Mensch im allgemeinen vielen Gruppen angehört. Eine Person (P) kann zum Beispiel wirtschaftlich zu dem oberen Mittelstand (oM) gehören - vielleicht handelt es sich um einen erfolgreichen Kaufmann. Er ist vielleicht Angehöriger einer kleinen Familie (F) von drei Personen, die Teil einer größeren Familiengruppe $(\mathrm{gF})$ ist, die in wenigen Städten des Ostens konzentriert ist. Diese größere Familiengruppe ist vielleicht die dritte amerikanische Generation irischer Vorfahren $\left(\mathrm{I}_{3} \mathrm{G}\right)$. Politisch ist der Betreffende möglicherweise Republikaner (Rep). Glaubensmäßig handelt es sich bei ihm etwa um einen Katholiken, und vielleicht hat er in seiner Kirchengruppe eine führende Stellung. (...)

Die längste Zeit seines Lebens handelt der Erwachsene nicht rein als Einzelwesen, sondern als Mitglied einer sozialen Gruppe. Jedoch sind die verschiedenen Gruppen, denen eine Person angehört, in einem bestimmten Augenblick nicht alle gleich wichtig. Bisweilen ist seine Zugehörigkeit zu der einen Gruppe beherrschend, bisweilen zu einer anderen. (...)" (Lewin, 1935, in LSK 1953, S. 105f)

Im letzten Abschnitt finden wir einen Hinweis auf Lewins differenzierte Sichtweise des Merkmals Zugehörigkeit.

Einen wichtigen Beitrag zur Frage der Bedeutung von Gruppe für das Leben des Einzelnen hat Metzger in seinem Werk „Psychologie in der Erziehung“ geleistet. Aus gestalttheoretischer und kritisch-realistischer Sicht hat er die Fälle gestörter Gruppen-Beziehungen und die Auswirkungen für die individuelle Entwicklung Betroffener systematisch zusammengestellt:

„Sofern Gruppen Real-Einheiten sind und der Mensch von Natur ein in Gruppen lebendes Wesen ist, befindet sich der einzelne nicht nur zu anderen einzelnen, sondern auch gegenüber Gruppen als ganzen, je nach 
dem Umständen, in einem , befriedigenden' oder, unbefriedigenden', das heißt in einem , ausgezeichneten' oder ,gestörten' Verhältnis. Und im zweiten Fall wird in ihm mehr oder weniger stark und ausdrücklich der Drang oder Trieb erwachen., aus dem gestörten in ein befriedigendes Verhältnis überzugehen. Typisch gestörte, und falls ihre Berichtigung unmöglich ist, Fehlentwicklungen auslösende Lagen sind für den Menschen unter anderem:

1. Das Ausgeschlossensein, das Bewußtsein, - trotz beliebig engen Zusammenlebens - nicht dazuzugehören, fünftes Rad am Wagen zu sein.

Folge: (...) aggressive Verwahrlosung, der Kampf gegen alle, (...) Antriebsverlust und Haltlosigkeit (...) (Lewin, 1953). Die Unmöglichkeit, sich anzuschließen, beispielsweise in einer fremdsprachlichen Umgebung oder bei Schwerhörigkeit, kann beim Erwachsenen paranoide Verfolgungsideen zur Folge haben (Schulte, 1924).

2. Hin- und hergerissen oder-gestoßen werden zwischen mehreren in Frage kommenden Gruppen: (...) der Pubertierende, der nicht mehr zu den Kindern, aber noch nicht zu den Erwachsenen paßt (Lewin, 1953); das Kind, das zwischen seinen geschiedenen Eltern hin- und hergerissen wird.

Folgen unter anderem: Reizbarkeit und Labilität.

3. Anomalien der gleichzeitigen Zugehörigkeit zu mehreren, einander überschneidenden Gruppen. Beispiel: bei Eheleuten die ungenügende Abgrenzung der neuen Gruppe gegen die Herkunftsfamilien (...). Folge: Wenn es nicht gelingt, die innerliche Trennung von der Herkunftsfamilie nachzuholen, Zerfall der Ehe.

4. Abweichungen von der Gleichwertigkeit und Gleichgeachtetheit.

a. Nach oben: Privilegierung, Verwöhnung, Bedienung.

Folgen u. a.: Wirklichkeitsverkennung, Vermessenheit, Gewissensverlust; dies auch noch bei Erwachsenen als Angehörigen von autoritären Cliquen, in denen sie sicher sein können, für keinen Verstoß zur Verantwortung gezogen zu werden.

b. Nach unten: Diskriminierung, Erniedrigung, Demütigung, Unterdrückung, Verhöhnung, Entrechtung.

Folgen: (...) Zweifel am eigenen Wert und Können; fortgesetzter Vergleich mit den anderen; weiterhin das Streben, die Unterlegenheit in Überlegenheit zu verwandeln: u.a. (...) Machtstreben, die Sucht, vollkommen zu erscheinen, Rechthaberei, Unfähigkeit, Kritik zu ertragen, bei gleichzeitiger Tendenz zu negativer Kritik, das heißt zur Abwertung der anderen; Unfähigkeit zu sachlicher Zusammenarbeit (u.a. Adler, 1912, Künkel, 1928, Dreikurs ,1969) 
5. Innerhalb einer Gruppe an falscher Stelle sein: zum Beispiel trotz mangelhafter Fähigkeit als „Führer“; (...) Als Folgen findet man die verschiedensten psychosomatischen Störungen (...). Im umgekehrten Fall, einer im Verhältnis zu den Fähigkeiten zu niedrigen Einstufung und verhinderten Aufstiegs: ,Versauern'.

6. Funktionslosigkeit: ,zu nichts nütze sein', für niemand da sein'. (...). Folgen: Bei sehr aktiver Veranlagung wird eine Funktion gesucht und gefunden, notfalls erfunden (zum Beispiel Vereinsgründung). Die Folgen sind anderenfalls: Schrumpfung der Interessen (...), Absinken des IQ.

7. Abweichungen von der gesunden Bewegungsfreiheit in einem weiten, aber begrenzten Entscheidungs- und Handlungsspielraum nach beiden Richtungen:

a. Fehlen aller Grenzen und jeden Haltes: Es gibt nichts, was man nicht darf, und man ,weiß nicht, was man soll'. Beispiel: die verzweifelte Lage eines Kindes, das nach dem Grundsatz des unbeschränkten Gewährenlassens (der ,permissiveness') behandelt wird und das ständig, tun soll, was es will', dem aber ohne äußere Anregung nichts mehr einfällt.

Folgen: Apathie oder Unfug.

b. Beengung der Bewegungsfreiheit, Unterbindung der Selbstbestimmung, Lahmlegen des Betätigungsbedürfnisses durch Versagung jedes Spielraums der Entscheidung und der Entfaltung. Beispiel: Erziehung nach dem ,Artigkeits'-Ideal.

Folgen: Neigung zu geheimer Bosheit und zu destruktiven Ausbrüchen schon bei geringfügigen Behinderungen noch im späteren Leben." (Metzger, 1976a, S. 45-47)

Zusammenfassend sei nochmals hervorgehoben, dass Lewin wie auch Metzger in ihren Schriften die Bedeutung der Gruppen für die Entwicklung des Individuums betonen:

„Das soziale Klima, in dem ein Kind lebt, ist für das Kind ebenso wichtig wie die Luft, die es atmet. Die Gruppe, zu der ein Kind gehört, ist der Boden, auf dem es steht. Sein Verhältnis zu dieser Gruppe und sein Rang in ihr sind die bedeutsamsten Faktoren für sein Sicherheits- oder Unsicherheitsgefühl. Kein Wunder, daß die Gruppe, von der die Person ein Teil ist, und die Kultur, in der sie lebt, zu einem hohen Grade ihr Verhalten und ihren Charakter bestimmen, welchen Raum freier Bewegung sie hat und wie weit sie mit einer gewissen Klarheit in die Zukunft blicken kann. Mit anderen Worten, sie bestimmen in einem wesentlichen Grade ihren persönlichen Lebensstil und die Richtung und Produktivität ihres Planens." (Lewin, 1939a, in LSK, 1953, S. 125) 
„Der Mensch ist ein soziales Wesen, das sich überhaupt nur als Glied einer Gruppe, als Teil eines, Wir', verwirklichen' kann. Die Gruppen, in denen der Mensch lebt, sind keine Fiktionen, keine Konstrukte, keine Gedankendinge. (Diese Annahme macht auch psychophysiologisch keinerlei Schwierigkeiten; vgl. Metzger 1969, 1971.) Sie sind genau so wirklich wie die Menschen, aus denen sie bestehen." (Metzger, 1975b, S. 22. Anmerkung MS: Die Klammereinfügung ist im Original eine Fußnote des Autors)

Im letzten Zitat argumentiert Metzger im Sinn des Kritischen Realismus: Es gibt demnach eine klare erkenntnistheoretische Basis für die soziale Bezogenheit des Menschen, aus der sich, wie in den folgenden Abschnitten zu zeigen sein wird, auch eine psychologisch begründete ethische Grundposition für das Zusammenleben mit anderen Menschen ableiten lässt. Der Einzelne nimmt in seiner phänomenalen Welt nicht nur andere einzelne Menschen, sondern ebenso Gruppen als reale Gegebenheiten wahr, mit denen er im Austausch steht, die ihn maßgeblich beeinflussen und für die er in seinem Handeln Mit-Verantwortung trägt.

\section{Gruppenführung und Gruppenatmosphäre: Demokratie als Bedingung für Freiheit und Kooperation}

Gordon W. Allport hebt in seiner Einleitung zur posthum von Gertrud Weiss Lewin herausgegebenen Schriftensammlung „Die Lösung sozialer Konflikte“ (deutsche Ausgabe 1953) die Verwandtschaft zwischen den Werken Kurt Lewins und John Deweys hervor:

„Es besteht eine überraschende Verwandtschaft zwischen dem Werk Kurt Lewins und dem Werk John Deweys. Beide sind der Meinung, die Demokratie müsse in jeder Generation neu erlernt werden und sie sei eine weit schwieriger zu erreichende und aufrecht zu erhaltende Form der sozialen Struktur als die Autokratie. Beide sehen die innere Abhängigkeit der Demokratie von der Sozialwissenschaft. Ohne Kenntnis der Gesetze der menschlichen Natur bei der Gruppenbildung und ohne Gehorsam gegen sie kann eine Demokratie keinen Erfolg haben. Und ohne Freiheit der Forschung und Theorie, wie sie nur in einer demokratischen Umgebung geboten wird, versagt die Sozialwissenschaft mit Sicherheit. Dewey, so können wir sagen, ist der hervorragende philosophische Exponent der Demokratie, Lewin ihr hervorragender psychologischer Exponent. Klarer als jeder andere hat er uns in konkreten, verwendbaren Begriffen gezeigt, was es heißt, ein demokratischer Führer zu sein und eine demokratische Gruppenstruktur zu schaffen." (Allport, 1953, S. 14f).

Tatsächlich hat sich Kurt Lewin in seiner Zeit in den USA intensiv dem Ziel gewidmet, einen methodischen Weg in der Psychologie zu finden, wie die „Merkmale und Kräfte des sozialen Feldes, in dem sich eine Person befindet", begrifflich 
angemessen beschrieben und quantitativ messbar gemacht werden können (vgl. Lewin, 1938, S. 141). Eine hervorragende empirische Ausgangbasis für dieses Vorhaben bildeten die Untersuchungen zur experimentellen Erzeugung unterschiedlicher sozialer Atmosphären in Gruppen mithilfe verschiedener Führungsstile, die Lewin und seine Mitarbeiter Ronald Lippitt und Ralph K. White in den Jahren 1937-1938 in der Iowa Child Welfare Research Station durchführten. Es lohnt sich auch nach Jahrzehnten, erneut einen Blick auf diese klassischen Experimente und ihre Ergebnisse zu richten. Sie gelten zwar „heute als die bekanntesten Lewin-Experimente" (vgl. Lück, 2009, S. 98f; Stützle-Hebel \& Antons, 2017, S. 8; Bogner, 2017, S. 283ff) und zählen nach wie vor zu den berühmtesten Experimenten der Sozialpsychologie. Zugleich wurden sie aber gerade in der akademischen Psychologie wie auch in der Erziehungswissenschaft der Nachkriegs-Bundesrepublik - in verkürzender Weise zitiert und missverstanden (Lück, 2009, S. 102f; Bogner, 2017, S. 283). Lück betont:

„Das Experiment steht (...) nicht in der Tradition positivistischer, empirisch-analytischer Forschung, obwohl es meist so dargestellt wird. Von Interesse für Lewin sind auch nicht einzelne Merkmale der Führung, auch nicht die ,Produktivität' der Führung, sondern das Klima, in dem ein Kind lebt." (Lück, 2015, S. 42)

Bogner vertritt die These, dass es Lewin

„(...) nicht oder nicht primär um den möglicherweise politisch oder biographisch motivierten Beweis der Überlegenheit eines bestimmten Führungsstiles ging, sondern darum, eine Möglichkeit zu finden, wie man die ,sozio-psychologischen' (...) Wirkungen bestimmter Milieus bzw. Atmosphären auf das Verhalten einzelner Individuen und ganzer Gruppen untersuchen könnte." (Bogner, 2017, S. 283)

Die in diesen Experimenten verwendete „Total Behavior Technique“, eine ungewöhnlich aufwändige und sorgsame quantitative und qualitative Datenerhebung vor, während und nach den experimentell erzeugten sozialen Situationen entsprach einer von der Gestalttheorie geprägten Methodologie (vgl. Lück, 2009, S. 16), die Lewin hier erstmals auf Gruppen anwandte:

„Der erste Zweck dieser Beobachtungstechnik ist es, so vollständig und einfühlsam wie möglich das gesamte Verhalten der Gruppe zu erfassen. Dies ist ein deutlicher Bruch gegenüber den üblichen Verfahren, die nur bestimmte, vorher festgelegte Symptome registrieren. Es ist vielmehr ein Versuch, die gleiche Methodologie des ,Gesamtverhaltens' (,total behavior' methodology) in der Sozialpsychologie anzuwenden, die sich in einer Anzahl von Untersuchungen zur Psychologie des Individuums (z.B. Dembos Studie über Ärger, Karstens Studie zur Sättigung sowie die Studie von Barker und Dembo über Frustration) als fruchtbar erwiesen hat. 
Der zweite Punkt, den wir betonen möchten, ist, dass exakte quantitative Daten wertlos werden, wenn man die Bedeutung aus dem Blick verliert, die diese einzelne Handlung innerhalb des ganzen Settings hat. Es ist daher äußerst wichtig, eine Charakterisierung der Gesamtsituation zu haben. Die notwendige quantitative Analyse (...) sollte immer im Kontext dieser größeren Gesamtheiten erfolgen." (Lewin \& Lippitt, 1938, S. 135)

Die Entstehungsgeschichte der Studien ist bei Lück (2009b, S. 99 und 2015, S. 42) und Bogner (2017, S. 283f) zusammengefasst. Das erste Experiment fand mit nur zwei Gruppen von je fünf 11-jährigen Jungen statt, in denen mittels unterschiedlicher Führung durch Lippitt selbst zwei unterschiedliche Atmosphären geschaffen wurden, eine „demokratische“ und eine „autokratische“. Eine zweite, aufwändiger geplante experimentelle Studie mit neuen Gruppen und mehreren neuen Führungspersonen, in der von vornherein Phasen wechselnder Führungsstile in allen Gruppen vorgesehen waren, schloss sich an. In dieser Studie wurde erstmals auch das dritte Führungsmuster, der „laissez-faire“-Stil², systematisch einbezogen.

Insgesamt trafen sich in diesen beiden Experimenten sechs sorgsam zusammengestellte Fünfergruppen von interessierten freiwilligen 10- und 11-jährigen Jungen jeweils mehrere Monate lang wöchentlich zu fest zusammengesetzten Spiel- und Bastelgruppen. Die Kinder fertigten Theatermasken, Wandmalereien, Seifenschnitzarbeiten, Gipsabdrücke ihre Füße und später auch Modellflugzeuge an. Dabei wurden sie von erwachsenen Leitern unterschiedlich geführt, wodurch verschiedene Gruppenatmosphären erzeugt wurden, „autokratisch“, „demokratisch“, und später auch im „laissez-faire“-Stil. Im zweiten Experiment wurden die unterschiedlichen Führungsstile nacheinander in den gleichen Gruppen angewandt: Nach jeweils 6 Wochen erfolgte ein Wechsel der erwachsenen Leitungsperson und damit zugleich auch jeweils der Führungsstil:

- In der autokratischen Atmosphäre wurden alle Entscheidungen vom erwachsenen Führer getroffen. Er bestimmte jeweils den unmittelbar anstehenden Arbeitsgang, und zwar so, dass die weitere Richtung in erheblichem Maß für die Gruppenmitglieder unklar blieb; er legte fest, wer mit wem zusammenarbeiten sollte; er lobte und kritisierte die einzelnen Gruppenmitglieder, ohne dafür Gründe zu nennen. Insgesamt verhielt er sich der Gruppe gegenüber distanziert. Sein Verhalten war eher unpersönlich als offen feindselig oder freundlich.

\footnotetext{
${ }^{2}$ Auf Lewins Betonung des Unterschiedes zwischen einer Atmosphäre des „laissez-faire“ und der Demokratie über die experimentelle Situation hinaus als Erziehungsform und gesellschaftliches Ideal weist Dirk Bogner (2017, S. 298f) mit Bezug auf Lewin (1936) und Lewin \& Lewin (1941) in prägnanter Weise hin: Demokratie als „voraussetzungsreiches“ Ideal, das wegen der notwendigen Partizipation an den Entscheidungsprozessen an das „Reifeniveau“ der so geführten Personen angepasst werden muss, um nicht in Anarchie abzugleiten. (vgl. auch Lewin, 1943, in LSK, S. 79)
} 
- In der demokratischen Atmosphäre wurden die Entscheidungen von der Gruppe gefällt, angeregt, ermutigt und betreut durch den Leiter. Wenn Rat gefordert wurde, nannte der Führer meist mehrere Lösungswege, unter denen einer auszuwählen war. Die Mitglieder konnten auch frei entscheiden, mit wem sie zusammenarbeiten wollten. Der Führer versuchte, „im Geiste“ Gruppenmitglied zu sein, arbeitete jedoch nicht aktiv mit. Er äußerte sachlich begründetes Lob oder Kritik, und zwar im allgemeinen der ganzen Gruppe gegenüber.

- In der „laissez-faire“-Atmosphäre hielt sich der Leiter völlig aus dem Gruppengeschehen heraus und nahm keinen Einfluss auf individuelles Tun oder Gruppen-Entscheidungen. Er stellte verschiedenes Arbeitsmaterial zur Verfügung, teilte mit, dass er für Informationen zur Verfügung stehe und gab dann auch nur auf Anfrage Rat. Er vermied es, das Verhalten und die Ergebnisse von Einzelnen und von der Gruppe positiv oder negativ zu bewerten; dennoch sollte sein Verhalten zur Gruppe möglichst die ganze Zeit über freundlich und nicht kühl distanziert sein. (vgl. Lück, 1996, S. 98ff/ Lewin \& Lippitt, 1938, S.133 / Lewin, Lippitt \& White, 1939, S. 279 / Lippitt \& White, 1947, S. 327f)

Während der Gruppentreffen wurden umfangreiche Beobachtungen zum Interaktions- und Sprachverhalten der Jungen angestellt. Die Zusammensetzung und Veränderung von Untergruppen wurde ebenso registriert wie der Grad des Interesses und der Beteiligung des einzelnen Gruppenmitglieds an den Gruppenaktivitäten von Moment zu Moment. Teilweise wurden Filmaufnahmen der Gruppentreffen angefertigt. Auch die eigenen Eindrücke der Gruppenleiter aus den „direkteren Kontakten mit den Kindern“ (Lewin \& Lippitt, 1938, S. 134) sowie Interviewaussagen der jugendlichen Gruppenteilnehmer im Anschluss an die jeweiligen Phasen gleicher Führung (Lewin, Lippitt \& White, 1939, S. 284f; Lippitt \& White, 1947, S. 330f) wurden in die Auswertungen einbezogen. Von den vielen Einzelergebnissen, die Lewin selbst und seine Kollegen in verschiedenen Arbeiten bereits ab 1938 dargestellt haben, werden hier die wichtigsten nochmals zusammengestellt:

In der demokratischen Gruppenatmosphäre wurden mehr kooperative Verhaltensweisen gezeigt. Es gab viel häufiger gegenseitiges Lob und Ausdruck freundlicher Gefühle, auch deutlich mehr Äußerungen des, Wir-Gefühls' und objektiver, sachlicher Einstellungen sowie konstruktiver Vorschläge zum weiteren Vorgehen, was auch zu einer "Überlegenheit der Gruppenergebnisse“ führte (Lewin, 1938, S. 143).

In den autokratisch geführten Gruppen fiel die Konstruktivität der Arbeit vor allem dann ab, wenn der Gruppenleiter für kurze Zeit den Raum verließ, während sie in der demokratisch geführten Gruppe „mit nur geringer Veränderung weiterging“ (ebd). 
In den laissez-faire-Gruppenphasen waren die Arbeitsergebnisse noch schlechter als in den autokratischen Atmosphären. Dies erklärt Lewin mit Ziellosigkeit:

„Im Gegensatz zu demokratischen und autokratischen Gruppen zeigt die Gruppe eines laissez faire, in der der Führer sich aus dem Spiel hält, nur gelegentliche Äußerungen einer Gruppenplanung oder langfristiger individueller Projekte. Die Arbeitsmoral einer solchen Gruppe ist im Vergleich zu der der demokratischen wie auch der autokratischen Gruppe sehr niedrig - ein Zeichen, wie wichtig für die Gruppenmoral klare Ziele sind. Nicht Ziele, die leicht erreicht werden können, sondern eine psychologische Zukunft mit Hindernissen und hohen Zielen führt zu einer hohen Moral“ (Lewin, 1942, in LSK, S. 170)

Vor allem unterschied sich das Ausmaß der gezeigten Feindseligkeit der Gruppenteilnehmer untereinander in den verschiedenen Gruppenatmosphären beträchtlich. So ergab sich in der ersten autokratisch geführten Gruppe ein deutlich höheres Aggressionsniveau als in der demokratisch geführten Gruppe: Streitigkeiten und verbale Feindseligkeiten zwischen den Jungen wurden ungefähr 30 mal häufiger registriert, aggressive Verhaltensweisen kamen $8 \mathrm{mal}$ häufiger vor. Im zweiten Experiment gab es wieder eine autokratisch geführte Gruppe mit ähnlich hohem Aggressionsniveau, während vier weitere Gruppen in autokratischen Führungsphasen extrem geringe Aggressionsraten, stattdessen aber apathische Verhaltensmuster zeigten. Die demokratisch geführten Gruppenphasen des zweiten Experiments zeichneten sich durch ein insgesamt moderates Aggressionsniveau aus; unmittelbar im Anschluss an vorherige „apathische Autokratie“ kam es jedoch vorübergehend zu höheren Aggressionsraten, die sich anschließend wieder normalisierten (vgl. Lewin, Lippitt \& White, 1939, S. 280). In der laissez-faireAtmosphäre wurde fast die gleiche Häufigkeit aggressiven Verhaltens beobachtet wie in der ersten autokratisch geführten Gruppe (vgl. ebd., S. 279).

Nach experimentell erzeugten „Störungen“ der Gruppensitzungen durch eine fremde Person („Hausmeister“), die jeweils in Abwesenheit des Leiters die Werke der Gruppe angriff, gab es ebenfalls charakteristische Unterschiede im Gruppenverhalten: die demokratisch geleiteten Gruppen tendierten zur gemeinsamen Verteidigung ihrer Arbeiten, die autoritär geführten Gruppen stattdessen zur Zerstörung der eigenen Basteleien (vgl. ebd., S. 289).

In ihren interpretierenden Kommentaren zu den Experimenten sprechen Lewin, Lippitt \& White von einer deutlich erhöhten atmosphärischen Grund-Spannung in der autokratischen Gruppe, die unter anderem mit einem erheblich eingeschränkten Raum freier Bewegung und der Rigidität der Gruppenstrukturen unter autokratischen Führungsbedingungen erklärt wird (Lewin, Lippitt \& White, 1939, S. 290ff). Interessanterweise verhielten sich die Jungen aber den autokratischen Führern gegenüber eher unterwürfig als offen aggressiv. 
Besonders beeindruckt war Lewin davon, dass es in der autokratisch geführten Gruppe des ersten Experimentes zweimal zu Sündenbock-Situationen kam, in denen sich die ganze Gruppe jeweils gegen eines ihrer Mitglieder verbündete und es zum Mittelpunkt der Aggression machte, so dass die betroffenen Opfer-Jungen schließlich nicht mehr zu den Treffen kamen. In demokratischen Atmosphären wurde solches "Scapegoating" nicht beobachtet.

Anhand der Filmaufnahmen konnte im zweiten Experiment außerdem die Veränderung der Atmosphäre in derselben Gruppe beim Wechsel des Führungsstils gezeigt werden:

„Nicht selten ist das dominante Merkmal der Autokratie nicht so sehr eine Atmosphäre der Feindschaft, sondern eher das der Primitivierung, des Mangels an Initiative und der Lustlosigkeit. Dies wird, glaube ich, sehr eindrucksvoll in einem unserer Filme gezeigt, mit dem der Wechsel der gleichen Kindergruppe von der Demokratie zur Autokratie dokumentiert wurde. Der letzte Tag der Demokratie zeigte die Kinder lebendig und bei intensiver Arbeit (unabhängig von der Anwesenheit oder Abwesenheit des Leiters), eine Atmosphäre der freundlichen Kooperation und eines gewissen Maßes an Konversation zwischen den Kindern. Es ist beeindruckend zu sehen, wie schnell während der ersten Stunde der Autokratie die Konversation zwischen den Kindern erstirbt. Wenn sich eine Frage ergibt, wird nur der Leiter kontaktiert; die Gesichter der Kinder werden weniger lebhaft, apathischer. Am fünften Tag der Autokratie zeigen die Filmaufnahmen, dass dieser Trend fest etabliert ist. Wenn der autokratische Leiter den Raum verlässt, wird die intensive Arbeit, die es in seiner Gegenwart gab, schnell verlassen und dies geschieht mit den gleichen Kindern, die in der Demokratie unabhängig produktive Arbeit mit Schwung geleistet haben." (Lewin, 1938, S.144)

Insgesamt ist zu resümieren, dass sich bei den Experimenten eine klare Überlegenheit des demokratischen Führungsstils zeigte, hinsichtlich der Gruppenbildung, der psychischen Befindlichkeit der Gruppenteilnehmer, der Produktivität und der beobachtbaren Aggression in den Gruppen. Auch hinsichtlich der individuellen Entwicklungsmöglichkeit im Rahmen der Gruppenteilnahme konstatiert Lewin:

„Bei unserem Experiment legte jeder einzelne in der Demokratie eine vergleichsweise größere Individualität an den Tag, da er trotz des unter allen herrschenden, Wir-Gefühls' oder vielleicht gerade deswegen einen gewissen Bereich für sich selber hatte. In der autokratischen Gruppe hatten dagegen die Kinder alle einen niedrigeren Rang ohne viel Individualität." (Lewin, 1939b, in LSK, S. 122)

Lewin hat vor der schnellen Verallgemeinerung der Ergebnisse gewarnt (1938, S.145), hat aber selbst in der Folgezeit oft auf diese Studien Bezug genommen, 
die nach Lück (1990, S. 153) auch „mehr oder weniger als Beitrag zur wissenschaftlichen Begründung von Demokratie dienen sollten."

\section{Erziehung und Bildung für die Demokratie}

Lewin beschäftigte sich - gut erklärbar, aber sicher nicht reduzierbar auf den Hintergrund seiner eigenen Migrationserfahrungen aus dem nationalsozialistischen Deutschland - intensiv und grundlegend mit der Demokratie. In vielen seiner in Amerika entstandenen Schriften kommt dies zum Ausdruck. Stellvertretend für viele ${ }^{3}$ sei hier aus der gemeinsam mit seiner Ehefrau, Gertrud Lewin, verfassten Arbeit „Demokratie und Schule“ (1941) zitiert:

„Die Grundlage der Demokratie besteht nicht im allgemeinen Stimmrecht, Mehrheitsentscheidungen und anderen organisatorischen Verfahrensweisen. Dies ist der technisch notwendige Überbau auf der Grundlage einer demokratischen Atmosphäre, ihre Konsequenz und Ausdrucksweise. Die Atmosphäre wird durch ein sehr subtiles Bekunden erzeugt, daß Menschen grundsätzlich gleich sind und Anrecht auf gleiche Achtung und Rücksicht haben." (Lewin \& Lewin, 1941, S. 286)

Besonders demokratische Erziehung lag Lewin am Herzen. Die gerade zitierte Publikation dient klar einer praktischen Anwendung und Umsetzung in der Schule. Lewin \& Lewin regen darin zum frühzeitigen Beginn demokratischer Praktiken und zur Gestaltung einer demokratischen Atmosphäre an, in der Kinder von Anfang an als denkende Personen mit eigenen Rechten behandelt werden; gerade deswegen seien sie auch bereit, Mitverantwortung für das soziale Leben in einer Gruppe zu übernehmen und Einschränkungen ihrer individuellen Freiheiten um der Gruppe willen zu akzeptieren:

\section{„Das Kind als denkende Person behandeln}

In demokratischer Atmosphäre wird das Kind von klein auf nicht als Objekt behandelt, sondern als Person; ihm werden Erklärungen und Begründungen für die Ereignisse in seiner Umgebung, besonders für notwendige Begrenzungen seiner Freiheit, gegeben; ihm wird das Recht gegeben, sich klar zu äußern, zu fragen und seine Sicht der Dinge auszusprechen. Ihm wird die Möglichkeit geboten, zu wählen und seine eigenen Entscheidungen zu treffen, wo immer dies vernünftigerweise möglich ist. Ein solches Kind entwickelt eine bessere affektive Basis für ein soziales Leben, ist darauf vorbereitet, Verantwortlichkeiten zu übernehmen, wenn es reif ist, mit anderen Kindern seines Alters zu spielen." (Lewin \& Lewin, 1941, S. 286)

\footnotetext{
3 Weitere Arbeiten Lewins zur Demokratie sind in dem 1953 in deutscher Sprache erschienenen Band „Die Lösung sozialer Konflikte“ versammelt; einige von ihnen und noch weitere hat Lück (2009) im Band „Kurt Lewin, Schriften zur Angewandten Psychologie“ erneut herausgegeben.
} 


\section{„Schranken der individuellen Freiheit}

Die Freiheit des Einzelnen ist nur eine Seite der demokratischen Lebensweise. Wenn sie uneingeschränkt ist, führt sie zu laissez-faire, zu Anarchie. Demokratie fordert Beschränkungen der individuellen Freiheit um der Gruppe willen. Das Kind, das in eine solche Gruppe hineinwächst, wird auf natürlichem Wege zu einer demokratischen Disziplin für das, Wohl aller' geführt (...). Kinder sind fähig, solche ,Beeinträchtigungen ihrer Rechte als Individuen' bereitwillig zu akzeptieren, wenn sie ihnen nicht wie von einer herrschenden Gruppe auf eine benachteiligte Minderheit auferlegt werden, sondern eher während eines Prozesses der Zusammenarbeit und des ,Gebens und Nehmens' entstehen." (Lewin \& Lewin, 1941, S. 286f)

„Die erfolgreichen Versuche in Richtung auf mehr demokratische Erziehung zeigen, wie viel die Schule beisteuern kann. Doch zeigen diese Versuche auch, daß der Umfang, in dem eine Erziehung für und durch Demokratie verwirklicht werden kann, davon abhängt, wie weit die Erwachsenen (selbst) fähig sind, in ihrer Welt Demokratie zu verwirklichen." (Lewin \& Lewin, 1941, S. 289f)

In den Schriften Wolfgang Metzgers spielt Demokratie und die Erziehung für die Demokratie seit den 1950er Jahren eine Rolle. Für ihn ist politische Bildung die Grundlage für eine verantwortliche Teilhabe im demokratischen Staat. Metzger sieht politische Bildung als besonders wichtiges Anwendungsgebiet des selbständigen Denkens im Sinn Wertheimers und Köhlers. (vgl. Metzger, 1980 / ausführlich in Soff, 2017, S. 176ff). Neben einer systematischen Förderung selbständiger, produktiver Denkprozesse im Rahmen des Unterrichts in möglichst vielen Schulfächern fordert er insbesondere die Einführung einer Gesprächs- und Diskussionskultur in der Schule, in der auf Machtausübung verzichtet wird, zugunsten einer sachlichen, vorurteilsfreien Argumentationsweise. Auch das fragende „den-Dingen-auf-den Grund-Gehen“ sollte im Unterricht Platz haben, ebenso wie die Anleitung, Informationen und Informationsquellen kritisch zu bewerten.

Schließlich betont Metzger, dass politische Bildung auch die Augen öffnen sollte für die „Unzulänglichkeiten und Vorläufigkeiten“ der Durchführung demokratischer Prozesse,

„für die gesellschaftlichen Mächte und Kräfte, die sich der Organisation des Staates ständig zu bemächtigen und sie in den Dienst ihrer speziellen Interessen und Machtgelüste zu stellen suchen. Der Bürger eines demokratischen Staates muß fähig sein, bestehende Mißstände ins Auge zu fassen, ohne seinen Glauben an die Idee und seine Zuversicht auf deren Verwirklichung zu verlieren“ (ebd. S. 45). 
Es geht nach Metzger also darum, zu unterscheiden, ob vorhandene Missstände unvermeidliche Folgeerscheinungen der demokratischen Ordnung oder ob sie gerade Verstöße gegen diese demokratische Ordnung darstellen. Nur auf dem Hintergrund dieser Unterscheidungen und in Kenntnis der Möglichkeiten, darauf in legitimer Weise Einfluss zu nehmen, kann sich demokratische Teilhabe mit dem Ziel der Verbesserung der staatlichen und gesellschaftlichen Ordnung entwickeln ${ }^{4}$.

Metzger plädiert dafür, die Begegnung von Schülern mit konkurrierenden Systemen und Gedankengebäuden nicht zu unterbinden und die Auseinandersetzung nicht zu scheuen. Trotz der konstatierbaren Unzulänglichkeiten im demokratischen Staat stellen für ihn die „Zusagen des Grundgesetzes“ die „einzigen wesentlichen Argumente für unser System“ dar - und nicht etwa der größere Wohlstand oder die beweglichere und glatter funktionierende Wirtschaft der damaligen Bundesrepublik Deutschland.

$\mathrm{Zu}$ diesen Zusagen des Grundgesetzes, die Schülern unbedingt vermittelt werden sollten, gehören u.a. die in den Artikeln 3, 4 und 5 verbürgten Rechte der Gleichberechtigung, der Religions- und Pressefreiheit, auf deren Wert in einer Demokratie als „soziale und politische Institutionalisierung der Toleranz" er besonders hinweist (Metzger, 1980, S. 48).

Sich auf diese differenzierte Weise mit der Demokratie als Staatsform und als „gute Form der Ordnung des Zusammenlebens" zu beschäftigen, war nicht nur für die 1940 er oder 1970er Jahre wichtig, sondern ist auch aktuell von größter Bedeutung. Und es scheint zunehmend wichtig, dass dies bereits in der Schule geschieht, als klar vorgetragener Bildungsinhalt mit Reflexions- und Diskussionsbedarf, darüber hinaus aber als gelebte Gleichberechtigung und Gleichgeachtetheit im „Miteinander in Verschiedenheit" in einer Schulklasse. Dazu gehört auch, dass sich Lehrerinnen und Lehrer nicht ,achselzuckend heraushalten“, wenn es z.B. um Diskriminierung und Mobbing unter Schülern geht, also um den sichtbaren Ausdruck von Vorurteilen.

\section{Von Minderheiten in Mehrheitsgesellschaften und vom Vorurteil zur Toleranz}

Mit der Begriffsbestimmung einer Gruppe über das Merkmal der Zugehörigkeit („Wir-Gruppe“) geht eine Begrenzung gegenüber „den anderen“, nicht als zugehörig Empfundenen, einher. Vor allem dann, wenn die Zugehörigkeit zur

\footnotetext{
${ }_{4}^{4}$ Dass der Versuch einer legitimen Einflussnahme auf gesellschaftliche und politische Entscheidungsprozesse mühsam und störungsanfällig bleibt und angesichts mächtiger Akteure mit einseitigen (z. B. rein wirtschaftlichen) Interessen auch als aussichtslos erlebt werden kann, ist ein Dauerthema in demokratischen Gesellschaften (vgl. dazu das aktuelle Beispiel „Gefährdungsbeurteilung zur arbeitsbedingten psychischen Belastung“, Ludborzs, 2017, S. 386ff, das durchaus Ähnlichkeit mit den von Lewin \& Lewin (1941, S. 287) konstatierten Mängeln in „pseudo-demokratischen“ Strukturen aufweist).
} 
Gruppe über den Faktor „Ähnlichkeit“ (übereinstimmende Merkmale, Homogenität) anstelle von „Interdependenz“ definiert wird, ist dies gleichzeitig eine kognitive Basis für die Bildung von Vorurteilen und Ausgrenzung (vgl Ioris, 2018, S. 10ff). Mit dem insgesamt komplexen und im menschlichen Zusammenleben allgegenwärtigen sozialpsychologischen Prozess der Vorurteilsbildung hat sich auch Metzger ausführlich beschäftigt (Metzger, 1976b, S. 52ff). In diesem Beitrag soll aber der Schwerpunkt darauf gelegt werden, wie Vorurteilen begegnet und was zu ihrer Überwindung getan werden kann.

Lewin hat nicht nur in Deutschland, sondern auch in Amerika, wohin er 1933 emigrierte, Vorurteile und Diskriminierung gegenüber der Minderheit der Juden erlebt. Seine letzten Lebensjahre waren u.a. davon geprägt, den Angehörigen (einer) seiner „Wir-Gruppe(n)“ Mut zuzusprechen und dem jüdischen Selbsthass (dem jüdischen Antisemitismus) entgegenzutreten. Gerade Lewins differenzierte Behandlung des Zugehörigkeitsgefühls (als Kriterium für das Vorhandensein einer Gruppe im Lebensraum des einzelnen Gruppenmitglieds) ist m.E. sehr nachvollziehbar vor dem Hintergrund seiner eigenen persönlichen Erfahrungen und vor allem seiner intensiven wissenschaftlichen Befasstheit mit der psychologischen Situation der Angehörigen von Minderheiten in einer Mehrheitsgesellschaft. Er behandelte in vielen Arbeiten die Frage, wie stark sich (vor allem junge) Juden im mehrheitlich nicht-jüdischen Amerika als Teil ihrer Minderheitsgruppe fühlten, oder wie stark sie - von der Übernahme gewisser Vorurteile der Mehrheitsgesellschaft gegen die eigene Gruppe beeinflusst - zum jüdischen Selbsthass neigten, also gerade keine Zugehörigkeit mehr wollten und sich sogar gegen die eigene Gruppe stellten (vgl. Lewin, 1935, 1939a, 1940, 1941a, 1941b, 1944, 1946a, 1946b, 1947). Dabei hat er stets feldtheoretisch fundiert argumentiert. Lewins persönliche Position ist eindeutig: er rät jüdischen Eltern zu einer mutigen und realistischen Erziehung zur deutlichen Zugehörigkeit und Identifikation mit der Herkunftsgruppe und zugleich zur Treue nach zwei Seiten (1940, S. 282). Diese Treue nach zwei Seiten führt gerade

„nicht zur ,Zweideutigkeit' (...), sondern zur Klarheit und der Überwindung der Randstellung von ethnischen Minderheiten, denn sie führt zum Bewusstsein, dass die Zugehörigkeit zu mehreren sozialen Gruppen ein Charakteristikum des Menschseins generell ist." (Lewin, 1940, S. 282; vgl. auch Bogner, 2017, S. 311)

Lewin spricht darüber hinaus ausdrücklich auch die Angehörigen der Mehrheitsgesellschaft mahnend an:

„In den letzten Jahren haben wir zu erkennen begonnen, dass die so genannten Minderheitenprobleme in Wirklichkeit Probleme der Mehrheit sind, dass das Rassenproblem das Problem des Weißen, das jüdische Problem das der Nichtjuden ist usw. (...). Die Diskriminierung, die die (...) einzelnen 
Personen erfahren, ist nicht gegen sie als Einzelne, sondern als Angehörige der Gruppe gerichtet, und nur dadurch, dass man ihre Selbstachtung als Gruppenangehörige auf das normale Maß hebt, lässt sich Abhilfe schaffen." (1946a, S. 258)

In der gleichen Arbeit thematisiert er auch die Interdependenz zwischen den verschiedenen Wir-Gruppen-Ebenen und tritt für eine Weitung des Blicks ein

„(...) zwischen dem örtlichen, dem nationalen und dem internationalen Bereich. Niemand, der auf dem Gebiet der Intergruppenbeziehungen arbeitet, kann sich der Tatsache verschließen, dass wir heutzutage in einer einzigen Welt leben. Ob wir an die Katholiken, die Juden, die Griechen oder die Schwarzen denken - jede Gruppe innerhalb der Vereinigten Staaten wird durch Ereignisse an anderen Stellen der Welt spürbar beeinflusst. Die Intergruppenbeziehungen hier in Amerika werden zu einem hohen Grade durch die Ereignisse im internationalen Bereich und besonders durch das Schicksal der kolonialen Völker geprägt werden.“ (1946a, S. 258f, Kursivhervorhebungen MS)

Aber wie kann sozialen Vorurteilen, z. B. gegen Minderheiten, entgegengewirkt werden? Lewin \& Grabbe (1945) behandeln diese Frage im Sinn eines Akkulturations- oder Umerziehungsprozesses und betonen die großen Schwierigkeiten, eine Veränderung von Vorurteilen zu erwirken. Schon die Veränderung der Kognition, eine Korrektur des vorhandenen Stereotyps, sei mit Hindernissen verbunden. Zusätzlich sei der Besitz korrekten Wissens in der Regel nicht ausreichend, „falsche Wahrnehmungen zu korrigieren“, da „Veränderungen in Gefühlen (...) nicht unbedingt Veränderungen der kognitiven Struktur" folgen (vgl. Lewin \& Grabbe, 1945, S. 228f). Bedeutsam für die Aufrechterhaltung oder den Abbau von Vorurteilen ist hingegen die soziale Atmosphäre, die das Individuum umgibt, und der dadurch auf den Einzelnen ausgeübte Anpassungsdruck:

„In jedem Bereich von Verhalten und Glauben übt die Gruppe einen starken Konformitätsdruck auf ihre einzelnen Mitglieder aus. Wir sind diesem Druck in allen Bereichen - politischen, religiösen, gesellschaftlichen - ausgesetzt, auch hinsichtlich unserer Ansichten darüber, was wahr oder unwahr, gut oder schlecht, richtig oder falsch, real oder irreal ist." (Lewin \& Grabbe, 1945, S. 225)

Mit der Schaffung einer neuen, „so genannten ,festen Gruppe‘, (...) der sich die Mitglieder zugehörig fühlen“, wird die Aussicht erhöht, dass die Gruppenmitglieder „ein neues System von Werten und Ansichten“ akzeptieren, die mit dieser Gruppenzugehörigkeit verbunden sind (vgl. ebd., S. 233). Allerdings kann eine Gruppe zur Veränderung von Vorurteilen nur dann mit Aussicht auf Erfolg eingesetzt werden, wenn es sich dabei um eine für alle Teilnehmer glaubwürdige Wir-Gruppe handelt, so dass ein Gefühl entstehen kann, „dass alle im 
gleichen Boot sitzen“, also „alle die gleichen Schwierigkeiten erlebt haben und die gleiche Sprache sprechen".

Aber selbst dann wird nach Lewin \& Grabbe das Aufzwingen eines neuen Systems von Werten und Ansichten, das zunächst die einfachste Lösung zu sein scheint, keinen Erfolg haben. Angesichts der Loyalität des Individuums zu älteren Überzeugungen wird Zwang sogar im Gegenteil zu Feindseligkeit gegenüber den neuen Werten (also zu Trotz und Widerstand) führen. Unter Bezug auf Allports „Axiom, dass man Menschen nichts beibringen kann, wenn diese sich gleichzeitig angegriffen fühlen" (Lewin \& Grabbe 1945, S. 234) und eine frühe Arbeit von Carl Rogers wird stattdessen eine Atmosphäre von Freiheit und Spontaneität in der Gruppe empfohlen, in der es auch möglich ist, alle Vorbehalte gegen etwaige Veränderungen offen anzusprechen.

„Es muss (...) hervorgehoben werden, dass, wenn Umerziehung die Schaffung eines neuen Über-Ich bedeutet, daraus notwendigerweise folgt, dass das angestrebte Ziel nicht erreicht werden kann, solange das neue Wertesystem nicht vom Individuum als etwas frei Gewähltes erlebt wird. Wenn sich das Individuum lediglich aus Angst vor Bestrafung statt der Stimme seines freien Willens und Gewissens folgend fügt, wird das neue Wertesystem, das es akzeptieren soll, in ihm nicht die Stellung eines ÜberIchs einnehmen, und seine Umerziehung bleibt unverwirklicht.

Daraus lässt sich schließen, dass soziale Wahrnehmung und Wahlfreiheit miteinander zusammenhängen. Seinem Gewissen zu folgen ist das gleiche, wie den wahrgenommenen situationsimmanenten Anforderungen zu folgen. Nur wenn das neue Wertesystem freiwillig akzeptiert wird, nur wenn es dem eigenen Über-Ich entspricht, werden jene Veränderungen in der sozialen Wahrnehmung auftreten, die, wie wir gesehen haben, eine Voraussetzung für eine Verhaltensänderung und damit für einen andauernden Effekt der Umerziehung sind.“ (ebd., S. 232).

Hier ergibt sich eine deutliche Parallele zur erziehungspsychologischen Position Wolfgang Metzgers (1975b, vgl. Soff, 2017, S. 96f). Metzger betont, dass Einsicht als Vorbedingung für freiwilligen Sachgehorsam nicht aufgrund von manipulativen Erziehungstricks erreicht werden kann, sondern allein dadurch, dass man sich - auf der Basis eines wechselseitigen Vertrauensverhältnisses - „an das Ich des Kindes", an das steuernde Zentrum einer Person wendet. Auch Lewin \& Grabbe heben hervor:

„Argumente, die logisch von einem Punkt zum nächsten gehen, können einen Menschen zwar in die Enge treiben. Aber in der Regel wird er einen Weg - wenn nötig einen sehr unlogischen Weg - finden, seine Ansichten zu behalten. Die hier besprochenen Aufsätze betonen immer wieder, dass bestenfalls kurzlebige Änderungen der Überzeugungen erreicht werden 
können, solange das Individuum nicht seine Feindseligkeit dem neuen Wertesystem als Ganzem gegenüber aufgegeben hat, und zwar in einem Maße, dass die Feindseligkeit zumindest in Offenheit übergegangen ist." (ebd., S. 233, Kursiv-Hervorhebung MS)

Das entspricht genau dem Erziehungsziel der Gestalttheorie: der Offenheit für die Forderungen der Lage.

In „Vom Vorurteil zur Toleranz“ bezieht sich Wolfgang Metzger ebenfalls auf Allports Arbeiten. Er weist darauf hin, dass bei sozialen Vorurteilen die unmittelbare Bekanntschaft mit Angehörigen der Gruppe der „Anderen“ „wirksamer (ist) als jede Belehrung durch Wort und Bild“. Dabei ist „die gemeinsame Verfolgung gemeinsamer Anliegen“ die wirksamste Möglichkeit (auch in der Schule), und zwar, „wenn sie so geplant wird, dass echt gemischte Arbeitsgruppen“ entstehen und die Aufgabe nur in wechselseitiger Interdependenz gelöst werden kann (Metzger, 1976, S. 95, nach Allport, letzte Ergänzung MS).

Allerdings wird der „Kampf gegen“ Vorurteile von Metzger nur als „fragwürdiger Notbehelf" angesehen, als Versuch einer nachträglichen Korrektur. Besser wäre es, dem Vorurteil von vornherein den Boden zu entziehen, also der Entstehung von Vorurteilen vorzubeugen. In diesem Sinn fordert er Maßnahmen auf unterschiedlichen Ebenen. Eine erste präventive Maßnahme besteht darin, Kindern gegenüber „gedankenlose Bemerkungen“ mit vorurteilsbehaftetem und diskriminierendem Inhalt über Andere zu unterlassen, um keinen „Unfrieden in das Herz der Kinder zu senken“. Darüber sollten Eltern und Erziehungspersonen aufgeklärt werden (vgl. ebd., S. 97). Zweitens ist die

„(..) Entstehung von Vorurteilen (...) besonders dort zu erwarten, wo eine Neigung zu einer blinden und kritiklosen Übernahme kaum oder gar nicht begründeter Meinungen besteht. Daraus ergibt sich die zweite Forderung, zu einer grundsätzlich kritischen - auch selbstkritischen - Haltung zu erziehen, bei der keine Meinung übernommen wird, ohne nach ihrer Herkunft und ihren Gründen zu fragen. Es gehört dazu auch die Pflege des Gefühls für die Sachverständigkeit des Verbreiters einer Meinung. (...) (Metzger, 1976b, S. 97)

Das knüpft an die schon erwähnte Forderung nach politischer Bildung als Sonderfall des selbständigen produktiven Denkens nach Wertheimer an. Und schließlich weist Metzger drittens darauf hin, dass ein Mensch

„um so starrer an seinen Meinungen fest (hält), je geringer seine Selbstsicherheit ist, je größer also sein Bedürfnis ist, von der Meinung der Gruppe gestützt zu werden und sich daher von der „Gruppennorm“ nicht allzu weit zu entfernen. Sein Bedürfnis, sich auf Kosten von anderen, in passenden Lagen auch durch die Erfindung von Sündenböcken, selbst 
zu erhöhen, ist umso größer, je mehr eine Erziehung zur Unselbständigkeit ihn verunsichert hat. Die Wirkung vervielfacht sich, wenn dieses Selbsterhöhungs- und Selbstbestätigungsbedürfnis unter sämtlichen Mitgliedern einer Gruppe verbreitet ist.

Das bedeutet, daß wir drittens die Erziehung zur Selbständigkeit und Selbstsicherheit, von der wir bisher nur geredet haben, entschlossen in Angriff nehmen müssen." (Metzger, 1976b, S. 98)

\section{Fazit und Ausblick: Was kann die Schule beitragen?}

Für die Frage, inwiefern Schulen mitwirken können bei Förderung von Toleranz und Mitverantwortung als Grundlage für die Teilhabe in einer demokratischen Gesellschaft, lassen sich nach der Beschäftigung mit den Werken Lewins und Metzgers einige Schlussfolgerungen formulieren:

1. Der erste und vielleicht wichtigste Beitrag kann in der Gestaltung einer guten Ordnung des Zusammenlebens in der Schule bestehen, in einer „Willkommenskultur" für alle Schülerinnen und Schüler, ungeachtet dessen, woher sie kommen und was sie persönlich mitbringen. Eine solche Ordnung, in der Zugehörigkeit und Gleichwertigkeit erlebt werden können, sollte darauf gerichtet sein, Ausgrenzungen zu vermeiden und möglichst kein Kind, keine(n) Jugendliche(n) „verloren gehen“ zu lassen. Das braucht einen klaren Rahmen, verlässliche Strukturen, aber vor allem eine zugewandt-interessierte alltägliche Präsenz der Lehrkräfte als Beziehungspersonen für die Einzelnen und das wache Gespür für die Atmosphäre in der jeweiligen gesamten Lerngruppe: Welchen Umgangston pflegen die Schüler untereinander? Und wie viel aggressive Spannung ist in einer Klasse? Wo bahnen sich etwa Mobbingprozesse an? (vgl. Schuster, 2013; Soff, 2017, Kap.V)

2. Demokratische Klassenführung mit sinnvoller Verantwortungs-Beteiligung für alle ist lernbar. Im Sinne Lewins ist dies zwar kein einfacher Vorgang, sondern an viele Bedingungen gebunden, da „die demokratischen Rechte und Verantwortlichkeiten des Kindes sich an sein Reifeniveau anpassen müssen“" (Lewin \& Lewin, 1941, S. 285; vgl. auch Bogner, 2017, S. 299f). Dies gilt umso mehr für Schulklassen, in denen sich Kinder und Jugendliche sehr unterschiedlicher Reifeniveaus befinden. Gebraucht werden dafür klare Konzepte (z.B. Klassenrat (vgl. Friedrichs, 2018; aber auch schon Dreikurs, 1968, 2007) oder die neuerdings propagierte Soziokratie (vgl. Breucker \& Errens, 2018)), aber vor allem prinzipielles Zutrauen in die Lernenden, die Duldung von Umwegen und die Prozessreflexionsbereitschaft seitens der Lehrerinnen und Lehrer, sowie eine klare institutionelle Verankerung und Unterstützung der demokratischen Führungsidee in der gesamten Schule. 
3. Unterricht, der das einsichtige Denken und die Lust auf Lernen fördert, statt sie zu zerstören, ist ein dritter und besonders zentraler Beitrag der Schule zur Demokratieförderung (vgl. Soff, 2017, Kap.VI). Insbesondere die Fähigkeit, sich mit neuen Problemen selbständig auseinanderzusetzen und die Fähigkeit und Bereitschaft, mit anderen zusammenzuarbeiten, in möglichst vielen Fächern und in Lernprojekten, wird nicht nur die kognitive, sondern auch die sozial-emotionale Entwicklung der Schülerinnen und Schüler günstig beeinflussen.

4. Eine direkte Anwendung des freien selbständigen Denkens ist es, mit jugendlichen Schülerinnen und Schülern im Unterricht über gesellschaftliche Themen zu sprechen, die sie interessieren und sie selbst betreffen. Eine solche Thematisierung dient zugleich der Entwicklung einer demokratischen Diskussionskultur. Vorausgesetzt, es handelt sich um eine hinreichend angstfreie Atmosphäre in der jeweiligen Gesprächsgruppe, können so auch immer wieder eigene Vorurteile gefunden, aufgedeckt und vielleicht gemeinsam dekonstruiert werden. Für diesen Vorschlag ist es allerdings notwendig, dass die Lehrpersonen nicht nur selbst hinreichend sachkundig in der jeweiligen Thematik sind, sondern auch die Fähigkeit entwickeln, zu einer sachlichen Aussprache angesichts kontroverser Meinungen anzuleiten (vgl. Soff, 2017, S. 179). Nützlich dafür ist sicher die Kenntnis kommunikativer Stilmittel, die Wolfgang Metzger in einer - überraschend aktuellen - Zusammenstellung hinterlassen hat. Es geht unter anderem darum, sich selbst und die Schülerinnen und Schüler

„mit den geläufigen ,falschen Beweismitteln’ bekannt und gegen sie stichfest zu machen:

- (...) das Ansprechen des Gefübls anstelle des Verstandes;

○ die unverfrorene Wiederholung einer unbewiesenen Behauptung, bis sie den Eindruck der Selbstverständlichkeit gewinnt;

- der Gebrauch von Etiketten (wie obsolet, hypermodern, rationalistische, mystisch, pazifistisch, militaristisch usw.), die ohne genauere Erfassung ihres Sinnes bei einem bestimmten Hörerkreis Zustimmung oder Ablehnung implizieren;

○ das einfache "bekanntlich", das auf den Hörer durch eine unbestimmte Zahl zuverlässiger Überzeugungsgenossen Eindruck zu machen sucht;

○ die Nennung von Autoritäten, gegen die der Gesprächspartner, wie man hofft, keinen Widerspruch wagen wird;

○ die Berufung auf - nicht näher gekennzeichnete - "neueste Forschungsergebnisse"; 
○ das Vorspiegeln einer Erklärung durch eine - möglichst eindrucksvolle - neue Benennung;

○ und dergleichen mehr." (Metzger, 1980, S. 37, Aufteilung in Zeilen MS)

Wer die Realität an Schulen kennt, weiß, dass die Umsetzung dieser Vorschläge alles andere als selbstverständlich ist. Nicht zuletzt ist darauf hinzuweisen, dass auch die Lehrerinnen und Lehrer verlässlichen Austausch und Rückhalt brauchen, um diesen anspruchsvollen pädagogischen und gesellschaftlichen Aufgaben gerecht zu werden. Eine Basis dafür stellt die Anerkennung und Ermutigung der einzelnen Lehrkräfte und eine im Kollegium gepflegte demokratische Führungskultur dar. Dafür zu sorgen, ist Aufgabe der Schulleitung. Angesichts vieler von außen an die Schulen herangetragener Vorgaben, die in den „Raum freier Bewegung“ der jeweiligen Schule eingreifen und Schulleitungen mitunter zu Ausführungsgehilfen sachlich wenig angemessener Verwaltungsvorschriften oder schulpolitischer Vorgaben machen, ist dies eine keineswegs leicht zu erfüllende, aber notwendige Forderung für die Balance der Lehrerinnen und Lehrer - und eine Chance für alle.

\section{Zusammenfassung}

In diesem Beitrag werden klassische Arbeiten von Kurt Lewin (1890-1847) und Wolfgang Metzger (1899-1979) zusammengestellt, die der Erziehung zu Demokratie und Toleranz gewidmet sind. Nach einer grundsätzlichen Erörterung zur Frage der Bedeutung der Gruppe(n) im Leben des Individuums werden zunächst die Experimente von Lewin, Lippitt \& White (1937/38) zusammenfassend gewürdigt, da sie die Auswirkungen von demokratischer vs. autokratischer vs. laissez-faire-Führung auf Gruppendynamik, Kreativität und Aggression verdeutlichen. Daraus ist zugleich Anschauungsmaterial für die Frage zu gewinnen, wie in aktuellen Schul-Situationen geführt werden kann, um Demokratie und Menschenrechte erlernbar und erlebbar zu machen. Lewins und Metzgers Texte enthalten darüber hinaus auch konkrete Hinweise, wie bestehenden Vorurteilen begegnet und dem Aufkommen von Vorurteilen präventiv entgegengewirkt werden kann. Abschließend werden auf der Basis des Präsentierten Vorschläge zum Beitrag von Schulen zur Förderung von Demokratie und Toleranz formuliert.

Schlüsselwörter: Demokratieförderung, Gruppenzugehörigkeit, Führungsstile, Politische Bildung, Erziehung zur Toleranz, Kurt Lewin, Wolfgang Metzger.

\section{Democracy as a Good Form of Coexistence}

\section{Education for Tolerance and Co-Responsibility in Freedom in the Scriptures of Kurt Lewin and Wolfgang Metzger}

\section{Summary}

In this article, classical writings of Kurt Lewin (1890-1947) and Wolfgang Metzger (1899-1979) concerning education for democracy and tolerance are presented. Starting 
with a definition of "group“ and the discussion of the meaning of groups for the individual life, especially the experimental studies of Kurt Lewin, Ronald Lippitt and Raph K. White in $1937 / 38$ on different group atmospheres under the influence of „democratic“ vs. „autocratic“ vs. „laissez faire“ leading style are presented again, in order to give an example for the meaning of atmosphere influences on personal development, creativity and aggression - all topics concerning actual school situations and the daily experience of pupils and teachers. The collection of writings concerning education for democracy and tolerance include some of Lewin's writings on minority problems and Metzger's fundamental thougts on political education as a special application of productive thinking in Wertheimer's sense. Some possibilities of how to change prejudices and to develop tolerance in Lewin's and Metzger's sense are presented. As a conclusion, some ideas for actual school life and possibilities to make pupils tolerant and able to participate actively in a democratic society are developed.

Keywords: Promotion of Democracy, Group Affiliation, Leadership Style, Civic Education, Education for Tolerance, Kurt Lewin, Wolfgang Metzger.

\section{Literatur:}

Allport, G.W. (1953). Einleitung. In G. Weiß Lewin (Hrsg.). Die Lösung sozialer Konflikte. Ausgewählte Abhandlungen über Gruppendynamik von Kurt Lewin. (S. 9-19). Bad Nauheim: Christian.

Bogner, D.P. (2017). Die Feldtheorie Kurt Lewins. Eine vergessene Metatheorie für die Erziehungswissenschaft. Wiesbaden: Springer VS.

Breucker, V. \& Errens, C. (2018). ...für echte Partizipation sorgen. Entscheidungen reflektiert und verantwortungsbewusst treffen. Pädagogik, 70/1, 13-15.

Dreikurs, R. (1968). Psychologie im Klassenzimmer. (2. Aufl.) Stuttgart: Klett

Dreikurs, R., Grunwald, B.B. \& Pepper, F.C. (Hrsg.) (2007). Lehrer und Schüler lösen Disziplinprobleme. Weinheim: Beltz.

Dunbar, R.I.M. (1998). The Social Brain Hypothesis. Evolutionary Anthropology. https://psych.colorado.edu/ tito/sp03/7536/Dunbar_1998.pdf (Zugriff am 22.02.2018)

Dunbar, R.I.M. (2009). The social brain hypothesis and its implications for social evolution. Annuals of Human Biology, 36/5, 562-572. https://doi.org/10.1080/03014460902960289 (Zugriff am 22.2.2018)

Edler, K. \& Schnack, J. (2017). Umgang mit Fundamentalismus und Intoleranz. Pädagogik 69/10, 6-8.

Friedrichs, B. (2018). ...für die Beziehung sorgen. Klasse leiten - aufgabenbewusst und partizipativ. Pädagogik $70 / 1,10-12$.

Horkheimer, M. (1953). Vorwort. In G. Weiß Lewin (Hrsg.). Die Lösung sozialer Konflikte. Ausgewählte Abhandlungen über Gruppendynamik von Kurt Lewin. (S. 7-8). Bad Nauheim: Christian.

Ioris, G. (2018). Sozialpsychologische Konzepte ethnischer Vorurteile im schulischen Kontext unter Berücksichtigung gestalttheoretischer Ansätze. Unveröff. Wissenschaftl. Hausarbeit, Pädagogische Hochschule Karlsruhe.

Lewin, K. (1935/1953). Psychosoziale Probleme einer Minderheitengruppe. In G. Weiß Lewin (Hrsg.). Die Lösung sozialer Konflikte. Ausgewählte Abhandlungen über Gruppendynamik von Kurt Lewin. (S. 204-221). Bad Nauheim: Christian.

Lewin, K. (1936/1953). Sozialpsychologische Unterschiede zwischen den Vereinigten Staaten und Deutschland. In G. Weiß Lewin (Hrsg.). Die Lösung sozialer Konflikte. Ausgewählte Abhandlungen über Gruppendynamik von Kurt Lewin. (S. 21-62). Bad Nauheim: Christian.

Lewin, K. (1938/2009). Experimente über autokratische und demokratische Atmosphären. In H.E. Lück (Hrsg.), Kurt Lewin, Schriften zur Angewandten Psychologie. Aufsätze - Vorträge - Rezensionen (S. 139-145). Wien: Krammer.

Lewin, K. (1939a/2009). Angesichts von Gefahr. In H.E. Lück (Hrsg.), Kurt Lewin, Schriften zur Angewandten Psychologie. Aufsätze - Vorträge - Rezensionen (S. 147-155). Wien: Krammer.

Lewin, K. (1939b/1953). Experimente über den sozialen Raum. In G. Weiß Lewin (Hrsg.). Die Lösung sozialer Konflikte. Ausgewählte Abhandlungen über Gruppendynamik von Kurt Lewin. (S. 112-127). Bad Nauheim: Christian. 
Lewin, K. (1939c/1963). Feldtheorie und Experiment in der Sozialpsychologie. In Cartwright, D. (Hrsg.), Kurt Lewin, Feldtheorie in den Sozialwissenschaften. Ausgewählte theoretische Schriften. (Lang, A., Lohr, W., Trans.) (S. 168-191). Bern: Huber.

Lewin, K. (1940/1982). Die Erziehung des Kindes. In C.-F. Graumann (Hrsg.), Kurt-Lewin-Werkausgabe, Bd. 6, (F.E. Weinert \& H. Gundlach (Hrsg.), Psychologie der Entwicklung und Erziehung (S. 267-283). Bern, Stuttgart: Huber, Klett-Cotta.

Lewin, K. (1941a/2009). Persönliche Anpassung und Gruppenzugehörigkeit. In H.E. Lück (Hrsg.), Kurt Lewin, Schriften zur Angewandten Psychologie. Aufsätze - Vorträge - Rezensionen (S. 171-177). Wien: Krammer.

Lewin, K. (1941b/2009). Selbsthass unter Juden. In H.E. Lück (Hrsg.), Kurt Lewin, Schriften zur Angewandten Psychologie. Aufsätze - Vorträge - Rezensionen (S. 157-169). Wien: Krammer.

Lewin, K. (1942/1953). Zeitperspektive und Moral. In G. Weiß Lewin (Hrsg.). Die Lösung sozialer Konflikte. Ausgewählte Abhandlungen über Gruppendynamik von Kurt Lewin. (S. 152-180). Bad Nauheim: Christian.

Lewin, K. (1943/1953). Der Sonderfall Deutschland. In G. Weiß Lewin (Hrsg.). Die Lösung sozialer Konflikte. Ausgewählte Abhandlungen über Gruppendynamik von Kurt Lewin. (S. 74-91). Bad Nauheim: Christian.

Lewin, K. (1944/1982). Jüdische Erziehung und Realität. In C.-F. Graumann (Hrsg.), Kurt-Lewin-Werkausgabe, Bd. 6 (F.E. Weinert \& H. Gundlach (Hrsg.), Psychologie der Entwicklung und Erziehung (S. 365-374). Bern, Stuttgart: Huber, Klett-Cotta.

Lewin, K., (1946a/2009). Aktionsforschung und Minderheitenprobleme. In H.E. Lück (Hrsg.), Kurt Lewin, Schriften zur Angewandten Psychologie. Aufsätze - Vorträge - Rezensionen (S.247-260). Wien: Krammer.

Lewin, K. (1946b/2009). Forschung über Minderheitenprobleme. In H.E. Lück (Hrsg.), Kurt Lewin, Schriften zur Angewandten Psychologie. Aufsätze - Vorträge - Rezensionen (S. 237-246). Wien: Krammer.

Lewin, K. (1946c/1982). Verhalten und Entwicklung als Funktion der Gesamtsituation. In C.-F. Graumann (Hrsg.), Kurt-Lewin-Werkausgabe, Bd. 6 (F.E. Weinert \& H. Gundlach (Hrsg.), Psychologie der Entwicklung und Erziehung (S. 375-448). Bern, Stuttgart: Huber, Klett-Cotta.

Lewin, K. (1947/1963). Gleichgewichte und Veränderungen in der Gruppendynamik. In D. Cartwright (Hrsg.), Kurt Lewin, Feldtheorie in den Sozialwissenschaften. Ausgewählte theoretische Schriften (S. 223-270). Bern: Huber.

Lewin, K. \& Grabbe, P. (1945/2009). Handeln, Wissen und die Übernahme neuer Werte. In H.E. Lück (Hrsg.), Kurt Lewin, Schriften zur Angewandten Psychologie. Aufsätze - Vorträge - Rezensionen (S. 223-235). Wien: Krammer.

Lewin, K. \& Lewin, G. (1941/1982). Demokratie und Schule. In C.-F. Graumann (Hrsg.), Kurt-Lewin-Werkausgabe, Bd. 6 (F.E. Weinert \& H. Gundlach (Hrsg.), Psychologie der Entwicklung und Erziehung (S. 285291). Bern, Stuttgart: Huber, Klett-Cotta.

Lewin, K. \& Lippitt, R. (1938/2009). Eine experimentelle Methode zur Untersuchung von Autokratie und Demokratie. Eine vorläufige Notiz. In H.E. Lück (Hrsg.), Kurt Lewin, Schriften zur Angewandten Psychologie. Aufsätze - Vorträge - Rezensionen (S. 131-138). Wien: Krammer.

Lewin, K., Lippitt, R. \& White, R.K. (1939). Patterns of aggressive behavior in experimentally created 'social climates'. Journal of Social Psychology, 20, 271-299.

Lippitt, R. \& White, R.K. (1947/1973). Eine experimentelle Untersuchung über Führungsstil und Gruppenverhalten. In C.F. Graumann \& H. Heckhausen (Hrsg.), Funkkolleg Pädagogische Psychologie. Grundlagentexte 1. Entwicklung und Sozialisation (S. 324-347). Frankfurt a. M.: Fischer.

Ludborzs, B. (2017). Gefährdungsbeurteilung zur arbeitsbedingten psychischen Belastung. Report Psychologie, 42/10, 386-390

Lück, H.E. (1991). Kurt Lewin - Demokrat und Reformer. Gestalt Theory 13/3, 148-158

Lück, H.E. (1996). Die Feldtheorie und Kurt Lewin. Eine Einführung. Weinheim: Beltz.

Lück, H.E. (2009a). Einführung. In H.E. Lück (Hrsg.), Kurt Lewin. Schriften zur angewandten Psychologie. Aufsätze - Vorträge - Rezensionen (S. 7-26). Wien: Krammer.

Lück, H.E. (2009b). Kurt Lewin. Eine Einführung in sein Werk. Weinheim: Beltz.

Lück, H.E. (2015). Kurt Lewin - der unbekannte Bekannte. In K. Antons \& M. Stützle-Hebel (Hrsg.), Feldkräfte im Hier und Jetzt. Antworten von Lewins Feldtheorie auf aktuelle Fragestellungen in Führung, Beratung und Therapie (S. 27-46). Heidelberg: Carl-Auer.

Metzger, W. (1975a). Was ist Gestalttheorie? In K. Guss (Hrsg.), Gestalttheorie und Erziehung (S.1-17). Darmstadt: Steinkopff (UTB).

Metzger, W. (1975b). Gibt es eine gestalttheoretische Erziehung? In K. Guss (Hrsg.), Gestalttheorie und Erziehung (S. 18-41). Darmstadt: Steinkopff (UTB). 


\section{GESTALT THEORY, Vol. 40, No.2}

Metzger, W. (1975c). Gestalttheorie und Gruppendynamik. In M. Stadler \& H. Crabus (Hrsg.), Wolfgang Metzger. Gestalt-Psychologie. Ausgewählte Werke aus den Jahren 1950 - 1982 (S. 210-226). Frankfurt a. M.: Kramer.

Metzger, W. (1976a). Psychologie in der Erziehung (3. Aufl.). Bochum: Kamp.

Metzger, W. (1976b). Vom Vorurteil zur Toleranz (2. Aufl.). Darmstadt: Steinkopff.

Metzger, W. (1980b). Politische Bildung aus der Sicht des Psychologen. In G.D. Hartmann (Hrsg.) Politische Bildung und politische Psychologie (S. 28-50) München: Fink.

Metzger, W. (2001). Psychologie. Die Entwicklung ihrer Grundannahmen seit der Einführung des Experiments (6. Aufl.). Wien: Krammer.

Schuster, B. (2013). Führung im Klassenzimmer. Disziplinschwierigkeiten und sozialen Störungen vorbeugen und effektiv begegnen - ein Leitfaden für Miteinander im Unterricht. Berlin: Springer VS.

Soff, M. (2017). Gestalttheorie für die Schule. Unterricht, Erziehung und Lehrergesundheit aus einer klassischen psychologischen Perspektive. Wien: Krammer.

Stützle-Hebel, M. \& Antons, K. (2017). Einführung in die Praxis der Feldtheorie. Heidelberg: Carl Auer.

Marianne Soff, (geb. 1956), Dr. phil., Diplom Psychologin, Psychologische Psychotherapeutin, Ausbildung in Gestalttheoretischer Psychotherapie. Seit 1998 Lehrtätigkeit als Akademische Oberrätin an der Pädagogischen Hochschule Karlsruhe, Schwerpunkte Erziehungspsychologie und Klinische Psychologie. Vorstandsmitglied der GTA und beratende Herausgeberin der Zeitschrift Gestalt Theory. Diverse Publikationen zu Gestalttheorie und Feldtheorie in verschiedenen Anwendungsfeldern, zuletzt "Gestalttheorie für die Schule. Unterricht, Erziehung und Lehrergesundheit aus einer klassischen psychologischen Perspektive“ (2017), Verlag Krammer, Wien, Tagebuchforschung, entwicklungspsychologische und erziehungspsychologische Beiträge.

Adresse: Pädagogische Hochschule Karlsruhe, Institut für Psychologie, Bismarckstr. 10, 76133 Karlsruhe, Deutschland

E-mail: soff@ph-karlsruhe.de 